\section{PHASE 1/2 STUDY TO EVALUATE PEPINEMAB IN COMBINATION WITH PEMBROLIZUMAB IN ADVANCED, RECURRENT OR METASTATIC HEAD AND NECK CANCER (KEYNOTE B84)}

${ }^{1}$ Elizabeth Evans*, ${ }^{1}$ Terrence Fisher, ${ }^{1}$ Crystal Mallow, ${ }^{1}$ Amber Foster, ${ }^{1}$ Ernest Smith, ${ }^{1}$ John Leonard, ${ }^{2}$ Marya Chaney, ${ }^{1}$ Maurice Zauderer. ${ }^{1}$ Vaccinex, Rochester, NY, USA; ${ }^{2}$ Merck and $\mathrm{Co}$, Kenilworth, NJ, USA

Background Immunosuppressive myeloid cells in the tumor microenvironment (TME) are a critical limitation to the efficacy of immune checkpoint inhibitors (ICIs) in patients with head and neck squamous cell carcinoma (HNSCC). Both semaphorin 4D (SEMA4D, CD100) and MDSCs are reported to play important roles in the growth and progression of HNSCC. Preclinical and clinical data demonstrated that antibody blockade of SEMA4D promotes tumor infiltration and activation of dendritic cells and CD8 $+\mathrm{T}$ cell, reverses immunosuppression, including attenuation of MDSC recruitment and function, and leading to enhanced efficacy of ICIs. ${ }^{1}{ }^{2}$ In a study evaluating pepinemab, a humanized SEMA4D blocking antibody, in combination with avelumab in patients with nonsmall cell lung cancer, the combination appeared to provide clinical benefit in patients with difficult to treat ICI-resistant and PD-L1-low tumors. ${ }^{3}$ Pembrolizumab is approved as first line therapy as monotherapy or in combination with chemotherapy in recurrent or metastatic (R/M) HNSCC, however not all patients respond to ICIs and require more effective treatments.

Methods KEYNOTE B84 (NCT04815720) is a multicenter, single-arm open-label study to evaluate the safety, efficacy, PK/ PD of pepinemab in combination with pembrolizumab in subjects with locally advanced, R/M HNSCC. Subjects with measurable disease per RECIST1.1 will be enrolled, including oropharynx, oral cavity, hypopharynx and larynx, and ECOG PS of 0 or 1 . Subjects who have received prior ICIs are excluded. This study will include a Safety Run-in phase $(n=3-$ 18) and a Dose Expansion (maximum $n=62$ ) phase. Pepinemab, which is well-tolerated in combination with other ICIs, will be evaluated starting with the highest intended dose of $20 \mathrm{mg} / \mathrm{kg}$, in combination with $200 \mathrm{mg}$ pembrolizumab, both administered intravenously every 3 weeks. The Dose Expansion phase will include an even distribution of subjects who have combined positive scores of $<20$ and $\geq 20$. The primary efficacy endpoint is ORR, and secondary endpoints include DOR, OS, PFS, as well as exploratory biomarker analysis. Pre- and on-treatment biopsies will be collected for evaluation of immune contexture in TME.

Results Screening has been initiated at several of a planned total of 18 sites. Multiplex immunohistochemistry (IHC) panels have been established to phenotype cells in the TME, including CD8+ T cells, DCs, MDSCs, Tregs, monocytes, macrophages.

Conclusions There remains a clear unmet need for more effective immunomodulatory treatment options to overcome immunosuppressive factors in the TME. The KEYNOTE B84 study will evaluate pepinemab as a potential treatment option to overcome resistance to and enhance activity of pembrolizumab in HNSCC.

Trial Registration NCT04815720

\section{REFERENCES}

1. Clavijo PE, Friedman J, Robbins Y, Moore EC, Smith E, Zauderer M, Evans EE, Allen CT. Semaphorin4D Inhibition Improves Response to Immune-Checkpoint
Blockade via Attenuation of MDSC Recruitment and Function. Cancer Immunol Res 2019 Feb;7(2):282-291.

2. Evans EE, Jonason AS Jr, Bussler $H$, Torno $S$, Veeraraghavan J, Reilly $C$, Doherty MA, Seils J, Winter LA, Mallow C, Kirk R, Howell A, Giralico S, Scrivens M, Klimatcheva K, Fisher TL, Bowers WJ, Paris M, Smith ES, Zauderer M. Antibody Blockade of Semaphorin 4D Promotes Immune Infiltration into Tumor and Enhances Response to Other Immunomodulatory Therapies. Cancer Immunol Res 2015 Jun;3(6):689-701.

3. Shafique MR, Fisher $\mathrm{TL}$, Evans $\mathrm{EE}$, Leonard JE, Pastore DRE, Mallow CL, Smith $\mathrm{E}$, Mishra V, Schröder A, Chin KM, Beck JT, Baumgart MA, Govindan R, Gabrail NY, Spira Al, Seetharamu N, Lou Y, Mansfield AS, Sanborn RE, Goldman JW, Zauderer M. A Phase Ib/ll Study of Pepinemab in Combination with Avelumab in Advanced Non-Small Cell Lung Cancer. Clin Cancer Res 2021 Jul 1;27(13):3630-3640.

Ethics Approval This study was approved by WIRB Copernicus Group's Ethics Board on 11Feb2021; approval number 20210250 .

http://dx.doi.org/10.1136/jitc-2021-SITC2021.434 